\title{
Pre-treatment of surface waters and wastewater by a hemodiafilter for online bacterial counting
}

Takahiro Fujioka ${ }^{a,{ }^{*}}$ My Thi Tra Ngo ${ }^{a}$, Ryo Makabe ${ }^{b}$, Sandrine Boivin ${ }^{a}$, Keisuke Ikehata ${ }^{c}$

\author{
${ }^{a}$ Graduate School of Engineering, Nagasaki University, 1-14 Bunkyo-machi, \\ Nagasaki 852-8521, Japan \\ ${ }^{b}$ R\&D division, Kyowakiden Industry Co., Ltd., 10-2 Kawaguchi-machi, \\ Nagasaki 852-8108, Japan \\ 'Ingram School of Engineering, Texas State University, 601 University Drive, \\ San Marcos, TX 78666-4684, U.S.A.
}

\section{Supporting Information}

* Corresponding author: Takahiro Fujioka, Email: tfujioka@nagasaki-u.ac.jp, Tel: +81 095819 2695, Fax: +81958192620 


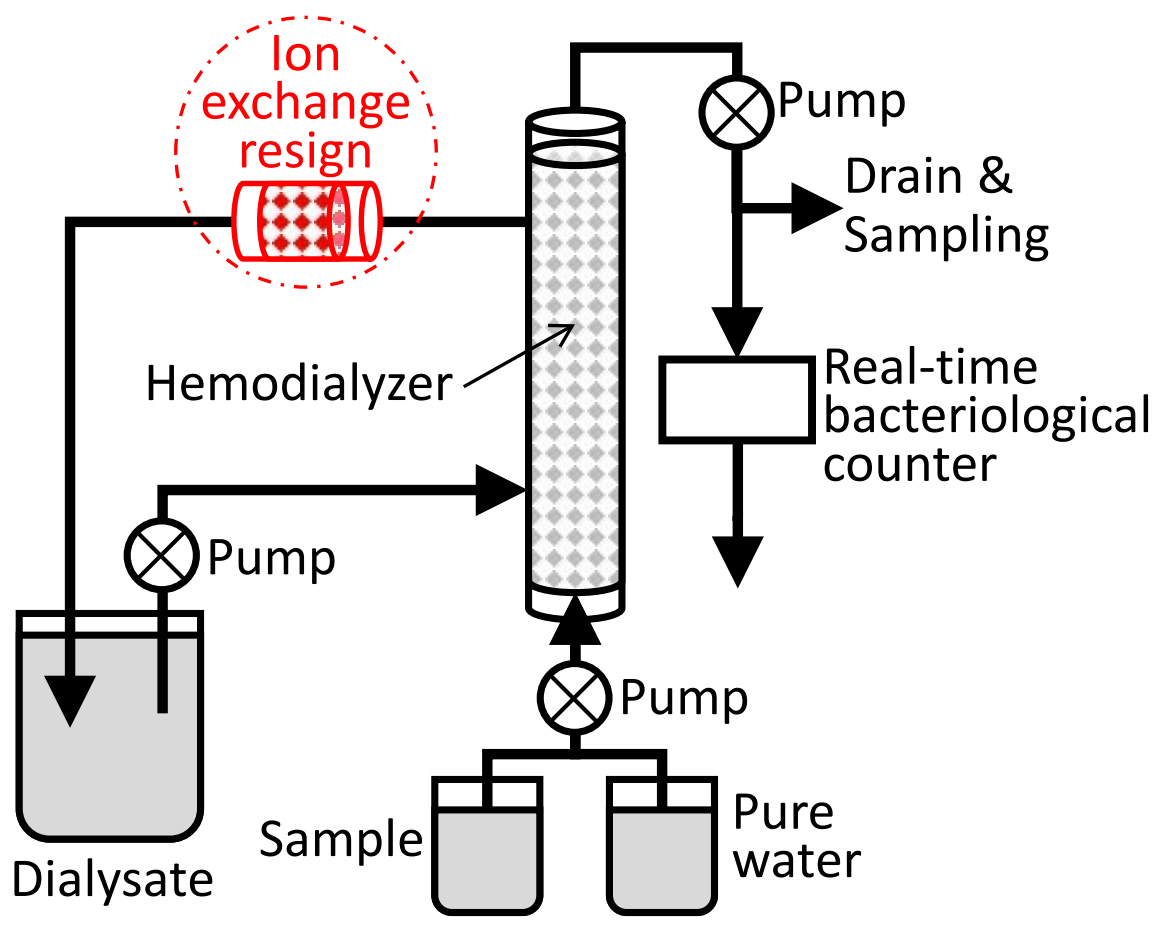

Fig. S1. Schematic diagram of the real-time bacteriological counter and online hemodiafilter pretreatment system equipped with an anion exchange resin column. 

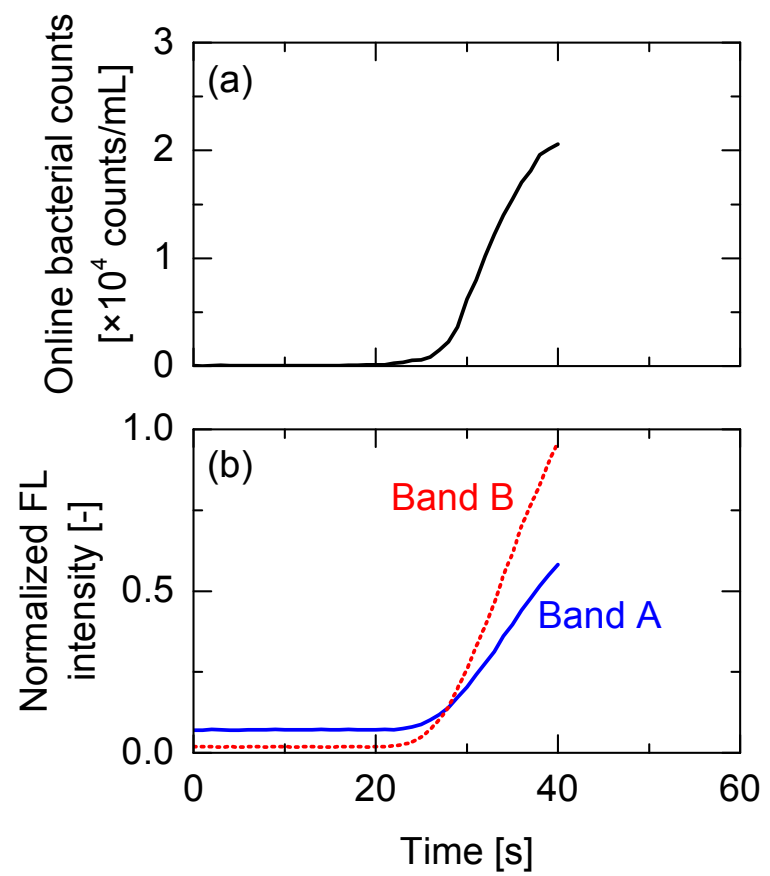

4

5 Fig. S2. (a) Online bacterial counts data for a river water without pre-treatment, (b) background 6 fluorescence (FL) intensity changes for samples in bands A and B for the real-time

7 bacteriological counter during analysis of the river water. Pure water in the feed was switched to 8 the river water at $0 \mathrm{~s}$, with data at $1-\mathrm{sec}$ intervals plotted as a line. The normalized background 9 fluorescence intensity of 1.0 is the maximum level measurable by the real-time bacteriological 10 counter. 


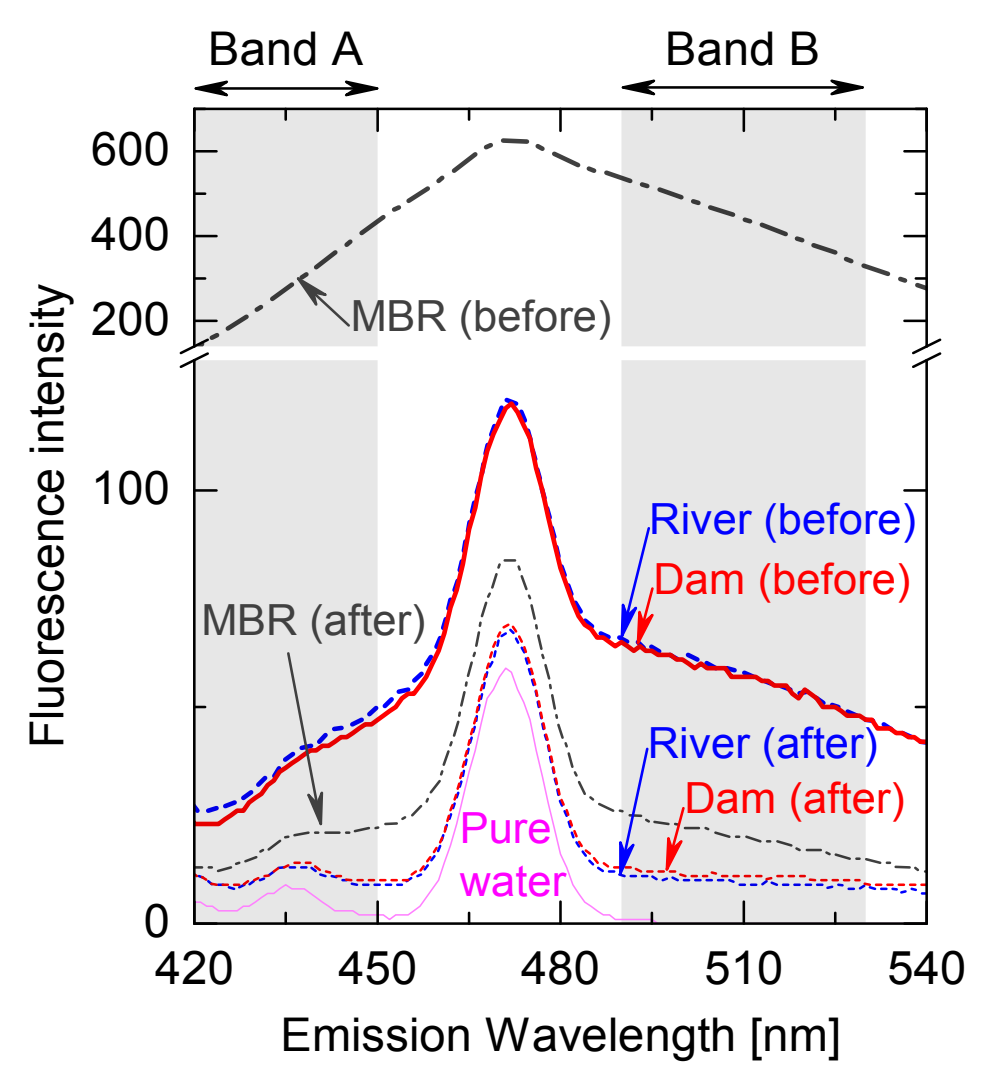

11

12 Fig. S3. Fluorescence spectra for the river water, dam water, and membrane bioreactor (MBR) 13 effluent at an excitation wavelength of $405 \mathrm{~nm}$ before and after the hemodiafilter pre-treatment. 14 These samples were collected after 20 min of online bacteria counting. 


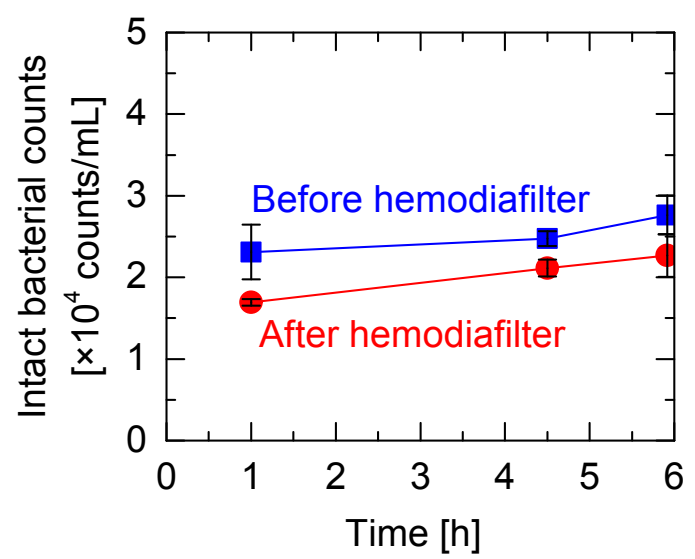

16 Fig. S4. Bacterial counts by epifluorescence microscopy (EFM) for the membrane bioreactor 17 (MBR) effluent over a 6-h duration after hemodiafilter pre-treatment involving an anion exchange 18 resign module. 